\title{
Incidence and prognostic significance of atrial fibrillation in acute myocardial infarction: the GISSI-3 data
}

F Pizzetti, F M Turazza, M G Franzosi, S Barlera, A Ledda, A P Maggioni, L Santoro, $\mathrm{G}$ Tognoni, on behalf of the GISSI-3 Investigators

\begin{abstract}
Background-Atrial fibrillation is the most common supraventricular arrhythmia in patients with acute myocardial infarction. Recent advances in pharmacological treatment of myocardial infarction may have changed the impact of this arrhythmia.

Objective-To assess the incidence and prognosis of atrial fibrillation complicating myocardial infarction in a large population of patients receiving optimal treatment, including angiotensin converting enzyme (ACE) inhibitors.

Methods-Data were derived from the GISSI-3 trial, which included 17944 patients within the first 24 hours after acute myocardial infarction. Atrial fibrillation was recorded during the hospital stay, and follow up visits were planned at six weeks and six months. Survival of the patients at four years was assessed through census offices.

Results-The incidence of in-hospital atrial fibrillation or flutter was 7.8\%. Atrial fibrillation was associated with indicators of a worse prognosis (age $>70$ years, female sex, higher Killip class, previous myocardial infarction, treated hypertension, high systolic blood pressure at entry, insulin dependent diabetes, signs or symptoms of heart failure) and with some adverse clinical events (reinfarction, sustained ventricular tachycardia, ventricular fibrillation). After adjustment for other prognostic factors, atrial fibrillation remained an independent predictor of increased in-hospital mortality: $12.6 \%$ v $5 \%$, adjusted relative risk (RR) $1.98,95 \%$ confidence interval (CI) 1.67 to 2.34. Data on long term mortality (four years after acute myocardial infarction) confirmed the persistent negative influence of atrial fibrillation (RR 1.78, 95\% CI 1.60 to 1.99 ). Conclusions-Atrial fibrillation is an indicator of worse prognosis after acute myocardial infarction, both in the short term and in the long term, even in an unselected population.
\end{abstract}

(Heart 2001;86:527-532)

Keywords: atrial fibrillation; acute myocardial infarction; prognosis

Department of

Cardiovascular

Research, Istituto di

Ricerche

Farmacologiche Mario

Negri, Milan, Italy

F M Turazza

M G Franzosi

$S$ Barlera

L Santoro

G Tognoni

Division of Cardiology, S Spirito Hospital, Casale Monferrato (AL), Italy

F Pizzetti

Division of Cardiology, Cervello Hospital,

Palermo, Italy

A Ledda

Centro Studi, ANMCO, Florence, Italy

A P Maggioni

Correspondence to: Dr F M Turazza, GISSI

Coordinating Centre,

Department of

Cardiovascular Research,

Istituto di Ricerche

Farmacologiche Mario

Negri, Via Eritrea 62, 20157

Milan, Italy

depcardio@irfmn.mnegri.it

Accepted 11 July 2001
Atrial fibrillation is one of the most common supraventicular arrhythmias in the setting of acute myocardial infarction, occurring in around $5-18 \%$ of all patients. ${ }^{1-7}$ The higher figures usually include individuals with pre-existing atrial fibrillation, and the probable incidence of new atrial fibrillation is closer to $5 \% .^{12}$

The arrhythmia develops for many different reasons, including left ventricular dysfunction with haemodynamic impairment, ${ }^{5-12}$ atrial ischaemia or infarction (particularly in patients with early onset atrial fibrillation in the course of acute myocardial infarction), ${ }^{13}$ right ventricular infarction, ${ }^{14}$ pericarditis, ${ }^{15}$ excessive release of catecholamines, chronic lung disease, acute hypoxia, drugs (for example, the use of sympathomimetic agents), and hypokalaemia. ${ }^{16}$

Atrial fibrillation is usually abrupt in onset and can cause rapid haemodynamic instability through one of three mechanisms: loss of the atrial component of the cardiac output; increased ventricular response rate with decreased diastolic filling time; or irregular ventricular filling. ${ }^{4}$

In many studies atrial fibrillation has been associated with advanced age,,$^{1-4717}$ congestive heart failure, poor left ventricular function, ${ }^{12} 71819$ and extensive myocardial infarction. ${ }^{4}$ Other associated conditions include mitral insufficiency, ${ }^{2}$ increased incidence of ventricular arrhythmias and right bundle branch block, ${ }^{3}$ and left bundle branch block. ${ }^{18}$ The occurrence of atrial fibrillation is not related to the site of the myocardial infarct. ${ }^{2} 315$

The relation of atrial fibrillation to outcome has been extensively investigated, and it is commonly considered a marker of poor prognosis. The occurrence of atrial fibrillation is associated with an increased hospital mortality ${ }^{20-23}$ though after adjustment for other variables known to affect prognosis - such as age, heart failure with cardiogenic shock, previous acute myocardial infarction, and ventricular arrhythmias-its independent effect appears to be somewhat reduced. It is possible, indeed, that the development of this arrhythmia merely reflects an overall increase in risk profile, as would be expected with large infarcts, decreased left ventricular function, and electrical instability.

Until very recently most studies of atrial fibrillation in relation to acute myocardial infarction have been relatively small and conducted in the prethrombolytic era. More recently, data from the large GUSTO-I study (global utilisation of streptokinase and tissue plasminogen activator for occluded coronary arteries) have been published, showing that atrial fibrillation in the setting of an acute myocardial infarct treated with thrombolysis independently predicted stroke and 30 day mortality. ${ }^{22}$ 
Current routine acute treatment for myocardial infarction includes not only aspirin, $\beta$ blockers, and thrombolytic agents, but also angiotensin converting enzyme (ACE) inhibitors. As shown by recently published clinical trials $^{23-25}$ and meta-analysis, ${ }^{26}$ this combined approach significantly reduces in-hospital and medium term mortality, left ventricular dysfunction and heart failure, myocardial damage extension, and ventricular arrhythmias. In this context, a recently published report by the TRACE (trandolapril cardiac evaluation) investigators $^{23}$ showed that in patients with a large acute myocardial infarct the occurrence of in-hospital atrial fibrillation is independently associated with an increase in hospital based and long term mortality. It is therefore relevant to reassess the incidence and prognostic significance of atrial fibrillation complicating acute myocardial infarction, in a substantial unselected population of patients receiving optimal treatment, including ACE inhibitors.

Despite the prevalence of atrial fibrillation, many issues still remain unresolved. The large GISSI-3 study allows a further exploration of this topic in the thrombolytic/ACE inhibitor era.

\section{Methods}

Data are derived from the GISSI-3 trial (gruppo Italiano per lo studio della sopravvivenza nell'infarto miocardico), a large clinical trial in which patients within 24 hours of acute myocardial infarction were randomised by telephone call or computer network system, working 24 hours a day, to receive oral lisinopril or no lisinopril and, according to a $2 \times 2$ factorial design, glyceryl trinitrate or no glyceryl trinitrate. The details of the design and the main results of the GISSI-3 study are provided in the original report. ${ }^{24}$

The entry criteria for the trial were: chest pain with ST segment elevation or depression of at least $1 \mathrm{~mm}$ in one or more peripheral leads of the ECG and/or at least $2 \mathrm{~mm}$ in one or more precordial leads; admission to a cardiac care unit within 24 hours from symptom onset; no clear indication or contraindication to the study treatments (oral lisinopril or intravenous and transdermal glyceryl trinitrate); Killip class $<4$; and absence of life threatening disorders.

\section{PATIENTS}

In all, 43047 patients were admitted to the 200 participating coronary care units; of these, 19394 (45\%) were randomised. Complete clinical data at the six week follow up were available for analysis for $18895(97.4 \%)$ of the randomised patients. Acute myocardial infarction was confirmed in 17944 patients, but our study population comprised 17749 patients. This was because we excluded from the final analysis 195 patients with chronic atrial fibrillation, defined as atrial fibrillation that was present on both admission and predischarge ECG records. (The purpose of the study was to assess the effects of new onset atrial fibrillation occurring during the peri-infarction period.)

All patients with no specific contraindications received the recommended drugs in the acute phase, according to defined clinical criteria: fibrinolytics were given in $72 \%$, intravenous $\beta$ blockers in $29 \%$, and aspirin in $85 \%$.

There were 8606 patients with confirmed acute myocardial infarction who underwent bidimensional echocardiography on discharge from hospital.

\section{END POINTS}

Investigators recorded all the relevant clinical events during the hospital stay and at clinical follow up visits after six weeks and six months from randomisation. On these occasions they were required to report if atrial fibrillation or flutter had occurred between randomisation and hospital discharge.

\section{STATISTICAL ANALYSIS}

The Pearson $\chi^{2}$ test for heterogeneity and the Mantel-Haenzel $\chi^{2}$ test for linear association (only for variables measured on an ordinal scale) were used to compare differences in atrial fibrillation incidence, observed in categorical variables.

Several Cox regression models, with the Mantel-Byar time to event correction, ${ }^{27}{ }^{28}$ were used to quantify the prognostic significance of atrial fibrillation with respect to in-hospital, six month, and four year mortality. The following covariates were included in the multivariate models: site of myocardial infarction, previous myocardial infarction, sex, age, history of hypertension, history of diabetes mellitus, Killip class, systolic blood pressure, history of angina, time from onset of symptoms, heart rate, in-hospital administration of anti-arrhythmic treatment, and the randomised treatment.

In assessing the prognostic significance of atrial fibrillation in patients discharged alive, no time-to-event correction was performed.

The proportionality assumption among hazards for the atrial fibrillation and no atrial fibrillation groups was tested using a flexible model-fitting approach involving restricted cubic spline function. ${ }^{29} 30$ This method allows one to examine the strength of the relation between hazard ratio and time (formulation and testing of a hypothesis on the effect of atrial fibrillation) and the shape of this relation (relative risk constant or not), without having to prespecify any functional form. The software used for the proportional assumption test and graphics is available on the world wide web. ${ }^{31}$

\section{Results}

INCIDENCE AND CLINICAL CHARACTERISTICS

The overall incidence of in-hospital atrial fibrillation was $7.8 \%$ (1386 patients). Among these patients, 319 had very early paroxysmal atrial fibrillation (that is, atrial fibrillation present on the admission ECG and absent on the predischarge ECG in patients discharged in sinus rhythm).

Patients who experienced atrial fibrillation were significantly older, had a worse Killip class at entry, and had a higher heart rate on their admission ECG. Other variables associated with this arrhythmia include previously treated hypertension, diabetes, female sex, and previous acute myocardial infarction (table 1 ). 
Table 1 Clinical characteristics of patients with or without atrial fibrillation $(A F)$

\begin{tabular}{|c|c|c|c|}
\hline Characteristic & Without $A F(n=16363)$ & With $A F(n=1386)$ & $p$ Value \\
\hline Women & 21.1 & 27.7 & $<0.0001^{\star}$ \\
\hline Age $>70$ years & 24.8 & 46.7 & $<0.0001^{\star}$ \\
\hline \multicolumn{4}{|c|}{ Time from symptom onset (hours) } \\
\hline$<3$ & 58.4 & 56.6 & \multirow{4}{*}{ NSt } \\
\hline$>3-6$ & 20.9 & 21.9 & \\
\hline$>6-12$ & 12.3 & 13.0 & \\
\hline$>12-24$ & 8.4 & 8.5 & \\
\hline \multicolumn{4}{|l|}{ Killip class at randomisation } \\
\hline 1 & 86.0 & 74.3 & \multirow{3}{*}{$<0.0001 \dagger$} \\
\hline 2 & 12.7 & 23.7 & \\
\hline 3 & 0.7 & 1.9 & \\
\hline \multicolumn{4}{|l|}{ Site of infarction } \\
\hline Anterior & 29.0 & 27.8 & \multirow{6}{*}{$<0.00001^{\star}$} \\
\hline Infero-posterior & 34.2 & 33.0 & \\
\hline Multiple location & 3.5 & 3.2 & \\
\hline Non-Q wave & 19.6 & 16.8 & \\
\hline Undefined & 9.7 & 15.1 & \\
\hline Not reported & 3.9 & 4.1 & \\
\hline \multicolumn{4}{|c|}{ Heart rate at randomisation (beats $/ \mathrm{min}$ ) } \\
\hline$<60$ & 11.5 & 9.3 & \multirow{4}{*}{$<0.00001 \dagger$} \\
\hline $60-79$ & 50.9 & 38.7 & \\
\hline $80-100$ & 33.3 & 39.2 & \\
\hline$>100$ & 4.2 & 12.8 & \\
\hline \multicolumn{4}{|c|}{ Systolic blood pressure at randomisation $(\mathrm{mm} \mathrm{Hg})$} \\
\hline $100-120$ & 39.5 & 36.7 & \multirow{3}{*}{$<0.03 \dagger$} \\
\hline $121-150$ & 46.2 & 46.8 & \\
\hline$>150$ & 14.4 & 16.6 & \\
\hline \multicolumn{4}{|l|}{ History } \\
\hline Previous MI & 13.2 & 15.4 & $<0.05^{\star}$ \\
\hline Previous angina & 34.2 & 34.8 & $\mathrm{NS}^{\star}$ \\
\hline Treated hypertension & 28.9 & 36.6 & $<0.001^{\star}$ \\
\hline Diabetes & 15.2 & 18.5 & $<0.01^{\star}$ \\
\hline
\end{tabular}

The $\mathrm{p}$ values $(2 \mathrm{p})$ are derived from $\chi^{2}$ tests:

${ }^{\star} \chi^{2}$ for heterogeneity.

$+\chi^{2}$ for trend.

MI, myocardial infarction.
Table 2 Non-study drugs given to patients with or without atrial fibrillation (AF) while in hospital

\begin{tabular}{llll}
\hline Drug & $\begin{array}{l}\text { Without AF } \\
(n=16363)\end{array}$ & $\begin{array}{l}\text { With AF } \\
(n=1386)\end{array}$ & p Value \\
\hline Fibrinolytics & 72.7 & 65.2 & $<0.001$ \\
Aspirin & 84.2 & 83.4 & NS \\
$\beta$ Blockers & 31.3 & 25.5 & $<0.0001$ \\
Digoxin & 5.7 & 32.5 & $<0.0001$ \\
$\begin{array}{l}\text { Oral antiarrhythmics } \\
\text { Oral anticoagulants }\end{array}$ & 5.6 & 39.8 & $<0.0001$ \\
$\quad$ (on discharge) & 3.1 & 4.3 & $<0.05$ \\
\hline
\end{tabular}

The data are percentages.

The $p$ values $(2 p)$ are derived from $\chi^{2}$ tests for heterogeneity. As expected, patients with atrial fibrillation were given significantly more digitalis and oral antiarrhythmic drugs. On the other hand, they were treated less frequently with fibrinolytics and intravenous $\beta$ blockers.

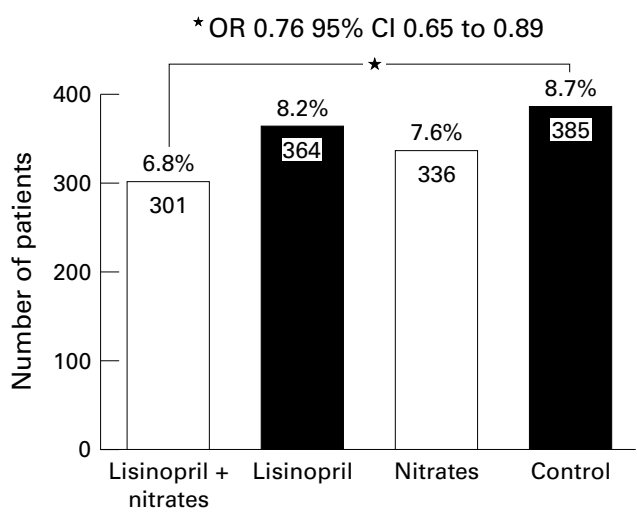

Figure 1 Distribution of atrial fibrillation $(A F)$ among randomised treatments: a significant $24 \%$ reduction of $A F$ incidence was observed in patients randomised to both lisinopril and nitrates compared with control patients.

The location of the infarct appeared less relevant, although patients with undefined location (that is, non-Q wave infarction with ST depression at entry) were significantly more likely to develop atrial fibrillation. The incidence of atrial fibrillation was higher in patients not treated with thrombolysis $(10.1 \%$ v $7.1 \%$; odds ratio (OR) $0.67 ; 95 \%$ confidence interval (CI) 0.59 to 0.75 ).

Finally, patients with in-hospital atrial fibrillation had worse left ventricular function, as shown by a significantly lower predischarge ejection fraction $(48.3 \% v 51.3 \%, \mathrm{p}<0.0001$, with $26.7 \% v 16.8 \%$ of patients having an ejection fraction $<40 \%$, $\mathrm{p}<0.0001$ ).

\section{TREATMENTS}

As shown in table 2, patients with atrial fibrillation were less often given the recommended non-study treatments-that is, fibrinolytics and $\beta$ blockers. On the other hand, patients with atrial fibrillation were more often being treated with digoxin, antiarrhythmic drugs, and oral anticoagulants on discharge.

With respect to randomised treatments, a $24 \%$ reduction (OR $0.76,95 \%$ CI 0.65 to 0.89 ) in the incidence of atrial fibrillation was observed in patients randomised to both lisinopril and nitrates compared with control patients (fig 1).

IN-HOSPITAL OUTCOMES

As shown in table 3, in-hospital death, early and late clinical congestive heart failure, sustained ventricular tachycardia, and ventricular fibrillation all occurred more often in patients with atrial fibrillation than in those without $(\mathrm{p}<0.001)$. No significant difference was observed in reinfarction rate, though this event appeared more common among patients with atrial fibrillation $(2.9 \% v 2.0 \%, \mathrm{NS})$. In this regard, no significant differences were shown between the two groups even when reinfarction and recurrent ischaemia were combined. Inhospital stroke rate was very low, and not significantly affected by atrial fibrillation.

The total hospital stay in patients discharged alive was significantly longer for those with atrial fibrillation than for those without (median stay $15 v 13$ days, $\mathrm{p}<0.0001$ ).

The unadjusted hospital mortality was higher in patients with atrial fibrillation than in those without (table 4). After adjustment for baseline differences, the hospital mortality remained significantly higher in patients with atrial fibrillation (RR 1.98, 95\% CI 1.67 to $2.34, \mathrm{p}<0.001)$.

The hospital mortality, but not the long term mortality, was significantly higher in patients developing "late" atrial fibrillation (after days $0-1)$ than in those developing early atrial fibrillation (occurring on days $0-1$ ). In the late group, hospital mortality was $18.9 \%$, compared was significant (OR 1.50, 95\% CI 1.08 to 2.08).

MEDIUM AND LONG TERM FOLLOW UP (SIX MONTH AND FOUR YEAR OUTCOMES)

Survival curves up to six months (fig 2) clearly show that a significant difference in favour of patients without atrial fibrillation is detectable with $13.3 \%$ in the early group. This difference 
Table 3 In-hospital events in patients with or without atrial fibrillation $(A F)$

\begin{tabular}{lccl}
\hline Event & $\begin{array}{c}\text { Without AF } \\
(n=16 \text { 363) }\end{array}$ & $\begin{array}{l}\text { With AF } \\
(n=1386)\end{array}$ & $p$ Value \\
\hline Clinical evidence of heart failure & 23.6 & 51.5 & $<0.0001$ \\
Congestive heart failure > 4 days & 3.8 & 12.1 & $<0.0001$ \\
Reinfarction + postinfarction angina & 13.8 & 15.3 & NS \\
Sustained ventricular tachycardia & 1.9 & 4.3 & $<0.0001$ \\
Ventricular fibrillation & 2.3 & 4.4 & $<0.0001$ \\
Death in hospital & 5.0 & 12.6 & $<0.0001$ \\
Stroke & 0.7 & 0.8 & NS \\
\hline
\end{tabular}

The data are percentages.

The $\mathrm{p}$ values $(2 \mathrm{p})$ are derived from $\chi^{2}$ tests for heterogeneity. The worse in-hospital outcome of the patients with atrial fibrillation is clearly apparent.

Table 4 Short term and medium term prognostic significance of atrial fibrillation developing in hospital

\begin{tabular}{lllll}
\hline & RR unadjusted & $95 \%$ CI & RR adjusted & $95 \%$ CI \\
\hline In-hospital mortality & 3.01 & 2.55 to 3.55 & 1.98 & 1.67 to 2.34 \\
Six month mortality & 2.93 & 2.40 to 3.57 & 1.81 & 1.48 to 2.23 \\
\hline
\end{tabular}

$\mathrm{CI}$, confidence interval; $\mathrm{RR}$, relative risk.

by the first few days; this increased during the first month but then remained unchanged over time.

The unadjusted six month mortality of patients discharged alive was higher in those with atrial fibrillation than in those without, regardless of the timing of the onset. The difference in favour of patients without atrial fibrillation was still evident after four years. After adjustment for baseline differences, the mortality in patients discharged alive remained

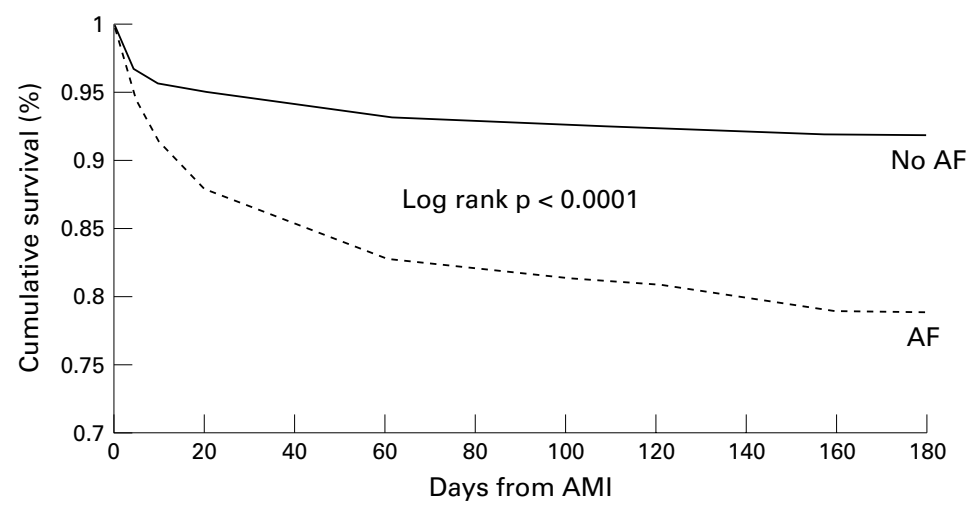

Figure 2 Six month survival of patients with or without the development of atrial fibrillation in hospital. AMI, acute myocardial infarction.

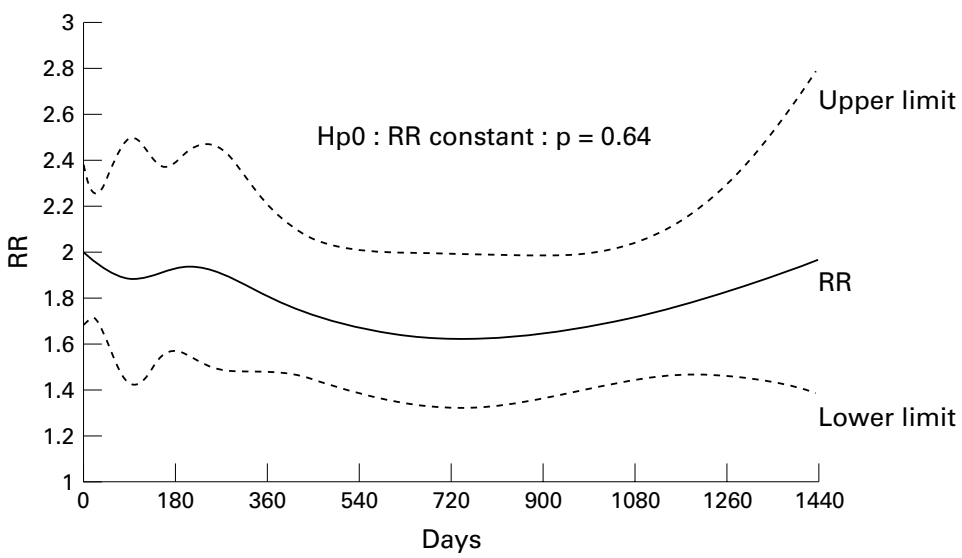

Figure 3 GISSI-3: four year follow up and relative risks (RR) of death from atrial fibrillation $(A F)$ versus no AF, giving the risk course over time for patients with in-hospital AF. The risk-time relation shows that even if the risk is higher during the very early postinfarction period $(R R=2)$ compared with the later stages, in the long term it appears constant and clearly not negligible.
Table 5 Long term (four years) independent predictors of mortality in patients discharged alive (Cox model)

\begin{tabular}{lll}
\hline & $\begin{array}{l}\text { Relative } \\
\text { risk }\end{array}$ & $95 \%$ CI \\
\hline Age >70 years & 2.93 & 2.70 to 3.18 \\
Killip class 3 & 2.12 & 1.56 to 2.89 \\
Atrial fibrillation & 1.78 & 1.60 to 1.98 \\
Killip class 2 & 1.73 & 1.58 to 1.90 \\
Left ventricular dilatation & 1.67 & 1.50 to 1.86 \\
Congestive heart failure $>4$ days & 1.65 & 1.43 to 1.90 \\
\hline
\end{tabular}

CI, confidence interval.

significantly higher in the group with atrial fibrillation, both at six months (RR 1.81, 95\% CI 1.48 to 2.23 ) and at four years (RR 1.78, $95 \%$ CI 1.60 to 1.99 ). The independent predictors of mortality in the long term are shown in table 5 .

Using a different multivariable model, and splitting our follow up into three different clinical periods, we observed that the risk course was clearly different between the hospital phase on the one hand and the post-acute (up to six months) and chronic phases (up to four years) on the other (fig 3).

INDEPENDENT PREDICTORS OF ATRIAL

FIBRILLATION

The most important predictor of atrial fibrillation was age $>70$ years (OR 2.82, 95\% CI 2.46 to 3.24). Other significant factors (in decreasing order) were raised heart rate at entry ( $>100$ beats $/ \mathrm{min}$, OR $2.19,95 \%$ CI 1.64 to 2.92 ), Killip class above 1 (OR $1.81,95 \%$ CI 1.55 to 2.11 ), and a history of hypertension (OR $1.34,95 \%$ CI 1.17 to 1.53 ).

\section{Discussion}

Atrial fibrillation is one of the most common supraventricular arrhythmias in the setting of acute myocardial infarction. It is also a marker of clinical and angiographic baseline features suggesting higher risk. ${ }^{22}$ In the GISSI- 3 trial, ${ }^{24}$ as in previous trials, ${ }^{20-22}$ old age was found to be the most important independent predictor of this arrhythmia.

Patients given randomised treatments (both nitrates and ACE inhibitors) were less likely to develop atrial fibrillation. It is hard to determine the precise aetiology of this arrhythmia, but the association with atrial fibrillation of Killip class above 1, a higher heart rate, and a lower ejection fraction-together with the trend towards a reduction in the incidence of atrial fibrillation in patients given nitrates and ACE inhibitorssuggests that haemodynamic impairment is the most likely mechanism. In fact in the GISSI-3 trial, patients allocated to the combination of lisinopril + nitrates not only had a lower mortality, but also a lower incidence of two other components of the combined end point directly related to haemodynamic impairment-clinical congestive heart failure and an ejection fraction less than $35 \%(8.3 \%$ in patients given lisinopril + nitrates, $v 9.2 \%$ in control, $9.5 \%$ with nitrates, and $9.0 \%$ with lisinopril). This is indirectly consistent with other studies which showed that patients given accelerated alteplase (greater than TIMI 3 flow rate) were less likely to develop 
atrial fibrillation, ${ }^{22}$ and that patients who developed atrial fibrillation had more unfavourable invasive haemodynamic variables than those who did not. ${ }^{512}$

HOSPITAL COMPLICATIONS

Patients with atrial fibrillation had a more complicated hospital course than those without. In our study, as well as in the GUSTO trial, reinfarction and recurrent ischaemia were more common, though not significantly so, among patients with atrial fibrillation. The lack of angiographic data did not allow an exhaustive interpretation of this finding, though it is likely that older and sicker patients, such as those with atrial fibrillation, had more extensive coronary disease.

Left ventricular dysfunction with a lower ejection fraction, clinical congestive heart failure, and cardiogenic shock were also more common in patients with atrial fibrillation. This is probably related, in a kind of vicious circle, to the impaired haemodynamics in these patients, where loss of atrial contraction leads to hypotension and possibly to more severe ischaemia. Patients with atrial fibrillation more often developed other arrhythmias, particularly ventricular tachycardia and ventricular fibrillation. Once again, among numerous possible explanations, the higher incidence of left ventricular dysfunction and congestive heart failure appears to play a pivotal role, although the more common use of digoxin and antiarrhythmic drugs could be important.

In our study, at variance with GUSTO-I, stroke incidence was not affected by atrial fibrillation. Overall stroke incidence was $0.7 \%$, the same in both groups, and clearly lower than observed in the GUSTO-I trial. Although there was a very low in-hospital stroke incidence in GISSI-3, it may well be that in sicker and more aggressively treated patients atrial fibrillation plays a role in increasing ischaemic stroke incidence, as shown in GUSTO-I.

MORTALITY

Our study, together with the recently published GUSTO and TRACE reports, ${ }^{22}{ }^{23}$ confirms that atrial fibrillation in the setting of acute myocardial infarction is associated with an increased in-hospital and long term mortality, probably owing to its association with worse left ventricular function. In the past, many studies ${ }^{8-12}$ have attempted to determine the relation between atrial fibrillation and mortality in patients with acute myocardial infarction, with conflicting results to some extent. For example, two recent large studies found a higher incidence of in-hospital and long term mortality in patients with than without atrial fibrillation. ${ }^{21}{ }^{21}$ In both studies, however, adjustment for other variables known to affect prognosis made the observed differences non-significant. The results differed for longer term mortality: in a multivariate analysis, one trial ${ }^{20}$ found that atrial fibrillation independently predicted long term mortality, whereas the other ${ }^{21}$ did not.

In our study, mortality was lower than previously reported. ${ }^{20}{ }^{23}$ Thus in the present study, in-hospital mortality in patients with and without atrial fibrillation was $12.6 \%$ and $5.0 \%$, respectively, versus $25.5 \%$ and $16.2 \%$ in the SPRINT (secondary prevention of reinfarction Israeli nifedipine trial) registry. ${ }^{20}$ This difference is a result of the improved treatment for acute myocardial infarction available in the 1990s, as well as differences in the populations studied-for example, all patients enrolled in GISSI-3 were haemodynamically stable, and the sickest subjects (that is, patients with persistent hypotension or cardiogenic shock) were excluded. The unadjusted and adjusted mortalities in GISSI-3 were significantly higher both in hospital and at the four year follow up among patients with atrial fibrillation than among those in sinus rhythm.

Our trial is the first capable of providing a long term follow up of a large population of patients with atrial fibrillation complicating acute myocardial infarction, and the data showing that atrial fibrillation developing in hospital is a sign of poor prognosis are of particular interest. The risk-time relation (fig 3) shows that though the risk is higher during the very early postinfarction period compared with the later stages, in the long run it appears constant, and definitely not negligible.

\section{CONCLUSIONS}

In the thrombolytic era atrial fibrillation remains a common and important complication of acute myocardial infarction. It identifies a group of patients who are older and sicker than their counterparts without atrial fibrillation. Such patients should be targeted for earlier and more aggressive treatment, because their overall prognosis-both short and long term - is significantly worse than in patients without atrial fibrillation. The management of patients developing atrial fibrillation after acute myocardial infarction should include intensive clinical surveillance, chronic anticoagulation, and cardioversion whenever possible.

GISSI is endorsed by the Associazione Nazionale Medici Cardiologi Ospedalieri (ANMCO) and Istituto di Ricerche Farmacologiche Mario Negri, Florence and Milan, Italy

1 Lofmark R, Orinius E. Supraventricular tachyarrhythmias in acute myocardial infarction. Acta Med Scand 1978;203: 517-20.

2 Helmers C, Lundman T, Mogensen L, et al. Atrial fibrillation in acute myocardial infarction. Acta Med Scand 1973;193:39-44.

3 Hunt D, Sloman G, Penington C. Effects of atrial fibrillation on prognosis of acute myocardial infarction. Br Heart $\mathrm{f}$ 1978;40:303-7.

4 Cristal N, Peterburg I, Szwarcberg J. Atrial fibrillation developing in the acute phase of myocardial infarction. Prognostic implication. Chest 1976;70:8-11.

5 Sugiura T, Iwasaka T, Ogawa A, et al. Atrial fibrillation in Sugiura T, Iwasaka T, Ogawa A, et al. Atrial fibrillation in

6 Klass M, Haywood LJ. Atrial fibrillation associated with Klass M, Haywood LJ. Atrial fibrillation associated with
acute myocardial infarction: a study of 34 cases. Am Heart acute myocardial in

F 1970;79:752-60.
7 Beck OA, Hochrein H. Atrial fibrillation and flutter as a complication of acute myocardial infarction. Dtsch Med Wochenschr 1976;101:1148-53.

8 Cristal N, Szwarcberg J, Gueron M. Supraventricular arrhythmias in acute myocardial infarction. Prognostic importance of clinical setting: mechanism of production. Ann Intern Med 1975;82:35-9.

9 Zoni Berisso M, Carratino L, Ferroni A, et al. The relation between supraventricular tachyarrhythmias and left ventricular dysfunction after acute myocardial infarction. $A c t a$ Cardiol 1988;43:689-701.

10 Sugiura T, Iwasaka T, Takahashi N, et al. Factors associated with atrial fibrillation in Q-wave anterior myocardial with atrial fibrillation in Q-wave anterio

11 Sugiura T, Iwasaka T, Takahashi N, et al. Atrial fibrillation in inferior wall Q-wave acute myocardial infarction. $A m \mathcal{F}$ Cardiol 1991;67:1135-6. 
12 Kobayashi Y, Katoh T, Takano T, et al. Paroxysmal atrial fibrillation and flutter associated with acute myocardial infarction: hemodynamic evaluation in relation to the development of arrhythmias and prognosis. Fpn $\operatorname{Circ} \mathcal{F}$ 1992;56:1-11.

13 Hod H, Lew AS, Keltai M, et al. Early atrial fibrillation during myocardial infarction: a consequence of impaired left atrial perfusion. Circulation 1987;75:146-50.

14 Rechavia E, Strasberg B, Mager A, et al. The incidence of atrial arrhythmias during inferior wall myocardial infarction with and without right ventricular involvement. $A m$ Heart f 1992;124:387-91

15 Liberthson RR, Salisbury KW, Hutter AM, et al. Atrial tachyarrhythmias in acute myocardial infarction. Am f Med 1976;60:956-60

16 Harrison DC. Atrial fibrillation in acute myocardial infarction. Significance and therapeutic implications. Chest 1976; 70:3-4.

17 McLean KH, Bett JN, Saltups A. Tachyarrhythmias in acute myocardial infarction. Aust N Z F Med 1975;5:3-6.

18 Flugelman MY, Hasin Y, Shefer A, et al. Atrial fibrillation in acute myocardial infarction. Isr F Med Sci 1986;22:355-9.

19 Siltanen P, Pohjola-Siktonen S, Haapakoski J, et al. The mortality predictive power of discharge electrocardiogram after first acute myocardial infarction. Am Heart $\mathcal{F}$ 1985;109:1231-7.

20 Behar S, Zahavi Z, Goldbourt U, et al. Long-term prognosis of patients with paroxysmal atrial fibrillation complicating acute myocardial infarction. SPRINT study group. Eur Heart F 1992;13:45-50.

21 Goldberg RJ, Seeley D, Becker RC, et al. Impact of atrial fibrillation on the in-hospital and long-term survival of patients with acute myocardial infarction: a communitywide perspective. Am Heart f 1990;119:996-1001.
22 Crenshaw BS, Ward SR, Granger CB, et al. Atrial fibrillation in the setting of acute myocardial infarction: the GUSTO-I experience. F Am Coll Cardiol 1997;30:406-13.

23 Pedersen O, Bagger H, Køber L, et al. The occurrence and prognostic significance of atrial fibrillation/flutter following acute myocardial infarction. TRACE study group. Eur Heart f 1999; 20:748-54.

24 Gruppo Italiano per lo Studio della Sopravvivenza nell'Infarto Miocardico. GISSI-3: effects of lisinopril and transdermal glyceryl trinitrate singly and together on 6 -week mortality and ventricular function after acute myocardial infarction. Lancet 1994;343:1115-22.

25 ISIS-4 (Fourth International Study of Infarct Survival) Collaborative Group. ISIS-4: a randomised factorial trial Collaborive Group. ISIS-4: a randomised factorial trial cute myocardial infarction. Lancet 1995;345:669-85.

26 ACE Inhibitor Myocardial Infarction Collaborative Group. Indications for ACE inhibitors in the early treatment of acute myocardial infarction. Systematic overview of individual data from 100000 patients in randomized trials. Circulation 1998;97:2202-12.

27 Cox DR. Regression models and life-tables. F R Stat Soc Ser B 1972;34:187-220.

28 Mantel N, Byar DP. Evaluation of response-time data involving transient states: an illustration using heart transplant data. F Am Stat Ass 1974;69:81-6.

29 Lee KL, Woodlief LH, Topol EJ, et al. Predictors of 30-day mortality in the era of reperfusion for acute myocardial inf(O) I 1659-68. 1659-68.

30 Hess KR. Assessing time-by-covariate interaction in proportional hazards regression models using cubic spline function. Stat Med 1994;13:1045-62.

31 Heinzl H, Kaider A, SAS Macro RCS. Available from: <www.akh-wien.ac.at/imc/biometrie/rcs.zip>, 1977.

\section{Electronic pages}

\section{eHEART: www.heartjnl.com}

The following electronic only articles are published in conjunction with this issue of Heart

(see also p 569)

Transseptal left heart catheterisation guided by intracardiac echocardiography

T Szili-Torok, GP Kimman, D Theuns, f Res, f R T C Roelandt, L F Fordaens

Objective - To develop a novel approach of transseptal puncture guided by intracardiac echocardiography and to assess its efficacy.

Methods-Transcatheter intracardiac echocardiography with a $9 \mathrm{MHz}$ rotating transducer was performed to guide transseptal puncture in 12 patients (mean age 43.1 years, range 31-68) who underwent radiofrequency catheter ablation of left sided accessory pathways. Initially, the echocardiography and transseptal catheters were placed adjacent to each other in the superior vena cava and were withdrawn to the level of the fossa ovalis.

Results-The successful puncture site was associated with visualisation of the fossa ovalis (12 patients, $100 \%$ ) and the aorta (12 patients, $100 \%$ ), tenting of the fossa (six patients, $50 \%$ ), penetration of the needle visualised by the ultrasound catheter (12 patients, 100\%), and echocardiographic contrast material applied in the left atrium (12 patients, $100 \%$ ). The characteristic jump of the needle onto the fossa ovalis was observed simultaneously with fluoroscopy and intracardiac ultrasound (12 patients, $100 \%$ ). All procedures were successful. There were no complications associated with the transseptal procedure. Conclusions-Intracardiac echocardiography is feasible to guide transseptal puncture. The optimal puncture site can be assessed by simultaneous detection of the characteristic downward jump of the transseptal needle onto the fossa ovalis by intracardiac ultrasound and fluoroscopy.

(Heart 2001;86:e11) www.heartjnl.com/cgi/content/full/ $86 / 5 / \mathrm{e} 11$

\section{Delayed post-traumatic tamponade together with} rupture of the tricuspid valve in a $\mathbf{1 5}$ year old boy $T$ Herbots, $P$ Vermeersch, $M$ Vaerenberg

With the increase in the number of high speed motor vehicle accidents, blunt, non-penetrating trauma to the heart has become an important health problem. An unusual case is reported of a 15 year old boy urgently referred with cardiac tamponade and a new systolic murmur four months after a car accident. The problems of the diagnosis and possible causes of late cardiac tamponade and tricuspid regurgitation following this type of accident are discussed. (Heart 2001;86:e12) www.heartjnl.com/cgi/content/full/ $86 / 5 / \mathrm{e} 12$

\section{Infected cardiac hydatid cyst \\ $M$ Ceviz, $N$ Becit, $H$ Koçak}

A 24 year old woman presented with chest pain and palpitation. The presence of a semisolid mass - an echinococcal cyst or tumour - in the left ventricular apex was diagnosed by echocardiography, computed tomography, and magnetic resonance imaging. The infected cyst was seen at surgery. The cyst was removed successfully by using cardiopulmonary bypass with cross clamp.

(Heart 2001;86:e13) www.heartjnl.com/cgi/content/full/ $86 / 5 / \mathrm{e} 13$ 